\title{
ON LOCALLY SOLUBLE PERIODIC GROUPS WITH CHERNIKOV CENTRALIZER OF A FOUR-SUBGROUP
}

\author{
by PAVEL SHUMYATSKY
}

(Received 19th August 1992)

Let $G$ be a locally soluble periodic group having a four-subgroup $V$. We show that if $C_{G}(V)$ is Chernikov then $G$ is hyperabelian-by-Chernikov, if $C_{G}(V)$ is finite then $G$ is hyperabelian.

1991 Mathematics subject classification (1985 Revision). 20F19, $20 \mathrm{~F} 50$.

\section{Introduction}

Centralizers play a very important role in locally finite group theory. In some cases we can deduce information about a locally finite group $G$ given only information about $C_{G}(V)$ for some finite subgroup $V$ of $G$ (see [10]). It is known now that if $G$ contains an element $v$ of prime power order with Chernikov centralizer then $G$ is almost locally soluble. The proof of this result in [11] depends on the classification of finite simple groups. In [2] Asar has proved that if $v$ above has order two then $G$ is almost soluble. This result does not depend on the classification but uses some essential parts of it.

In this article we turn our attention to groups $G$ containing a four-subgroup $V$ such that $C_{G}(V)$ is Chernikov. These groups can be simple. Indeed, the example of an infinite simple locally finite group having a four-subgroup with Chernikov centralizer is provided by $\operatorname{PSL}(2, F)$ where $\mathbf{F}$ is an infinite locally finite field of odd characteristic. A locally soluble periodic group with Chernikov centralizer of the four-subgroup also can be non-soluble. To show this we use an example from [12].

Let $p$ be an odd prime and let $t$ denote the largest odd divisor of $p-1$. Let $G_{k}$ be the group formed by the matrices

$$
A=\left(\begin{array}{cc}
u+p a & p b \\
p c & v+p d
\end{array}\right)
$$

of determinant 1 , where $a, b, c, d, u, v$ lie in the ring of residue classes $\left(\bmod p^{k+1}\right)$ and $u v=u^{z}=1(\bmod p)$. Then $G_{k}$ is of derived length $m$ or $m+1$ where $m$ is the least integer such that $2^{m} \geqq k+1$. Let $\alpha_{k}$ and $\beta_{k}$ be the elements of Aut $G_{k}$ such that $A^{a_{k}}=\left(A^{-1}\right)$ and $\left(\begin{array}{ll}a_{1} & a_{2} \\ a_{3} & a_{4}\end{array}\right)^{\beta_{k}}=\left(\begin{array}{cc}a_{1} & -a_{2} \\ -a_{3} & a_{4}\end{array}\right)$. It is not difficult to verify that $\left\langle\alpha_{k}, \beta_{k}\right\rangle$ is a four-group acting fixed-point-freely on $G_{k}$. Let $G$ be the direct product of groups $G_{k} ; k=1,2, \ldots$. Then $G$ 
admits a fixed-point-free four-group of automorphisms $V$. Clearly, the split extension of $G$ by $V$ is the required group.

In this paper we prove:

Theorem. Let $G$ be a periodic locally soluble group and $V$ a four-subgroup of $G$.

(i) If $C_{G}(V)$ is Chernikov then $G$ is hyperabelian-by-Chernikov.

(ii) If $C_{G}(V)$ is finite then $G$ is hyperabelian.

We recall that a group is said to be hyperabelian if it has an ascending invariant series with abelian quotients. In [14] the author proved that a locally finite group admitting a fixed-point-free four-group of automorphisms is hyperabelian. In the present paper we use some important technic and ideas of [14].

\section{Lemmas}

Lemma 1. Let $G$ be a locally soluble group containing a hyperabelian subgroup of finite index. Then $G$ is hyperabelian.

Proof. Clearly, $G$ contains a normal hyperabelian subgroup $H$ of finite index. Suppose $G$ is not hyperabelian. Then there exists a quotient $G / R$ which does not possess an abelian normal subgroup. Let the images of $G$ and $H$ in $G / R$ be denoted again by $G$ and $H$ respectively. Let $A$ be a nontrivial normal abelian subgroup of $H$. Then $\left\langle A^{G}\right\rangle$ is the product of finitely many abelian normal subgroups of $H$ and so is nilpotent. Thus $Z\left\langle A^{G}\right\rangle$ is a nontrivial normal abelian subgroup of $G$.

Lemma 2. Let $\pi$ be a set of primes, $G$ a locally finite $\pi^{\prime}$-group acted on by a finite $\pi$-group $V$.

(i) If $N$ is a normal $V$-invariant subgroup of $G$ then $C_{G / N}(V)=C_{G}(V) N / N$.

(ii) $[G, V]=[G, V, V]$.

(iii) $G=[G, V] C_{G}(V)$.

Proof. Each of these facts follows immediately from the corresponding finite case [7].

Lemma 3. Let $G$ be a locally finite group acted on by an involutory automorphism $v$ in such a manner that $C_{G}(v)$ is Chernikov. Let $\mathrm{O}(G)$ be the largest normal 2'-subgroup of $G$, and let $F$ be a divisible abelian 2-subgroup of $G$ such that $x^{v}=x^{-1}$ for every $x \in F$. Then $[O(G), F]=1$.

Proof. Since $F \subseteq[G, v]$, we have $[O(G), F] \subseteq[G, v]$ so by $[2]$ and $[9$, Theorem B] $[O(G), F, F]$ is Chernikov whence, by $[4],[O(G), F, F, F]=1$ and, by the preceding lemma, $[0(G), F]=1$, as required.

Throughout the remaider of this section let $G$ stand for a locally finite 2 '-group, $V$ an 
elementary group of automorphisms of order $2^{n}$. Let $V_{1}, V_{2}, \ldots, V_{2} n_{-1}$ be the set of maximal subgroups of $V, G_{i}=C_{G}\left(V_{i}\right), J_{i}=\left\{x \in G_{i} ; x^{v}=x^{-1}\right.$ for $\left.v \in V-V_{i}\right\} ; 1 \leqq i \leqq 2^{n}-1$.

Lemma 4. Let $n=2$ and $v_{i}$ be the involution from $V_{i} ; 1 \leqq i \leqq 3$. Suppose that $x, y \in G$ and $v_{3}$ sends $x$ into $x^{-1}$. Then

(i) there exists a unique pair of elements $a \in J_{1}, b \in J_{2}$ such that $x=b a b$;

(ii) there exist elements $y_{1} \in G_{1}, y_{2} \in G_{2}, y_{3} \in G_{3}$ such that $y=y_{1} y_{2} y_{3}$.

Proof. See [15, Lemmas 1.4 and 1.6].

Remark. Suppose that the $x$ above is conjugate in $G$ to some element of $G_{1}$. Then $b \in G^{\prime}$.

Indeed, let $\bar{z}$ denote the image of $z$ in $\bar{G}=G / G^{\prime}$. Then $\bar{x} \in C_{\bar{G}}\left(v_{1}\right)$, whence $\bar{x}=\bar{a}$. So $b=1$ as required.

Lemma 5. If $G=[G, V]$ then

(i) $G=\left\langle J_{i} ; 1 \leqq i \leqq 2^{n}-1\right\rangle$;

(ii) $C_{G}(V)$ is generated by its subgroups $C_{G}(V) \cap\left\langle J_{i}\right\rangle, 1 \leqq i \leqq 2^{n}-1$.

Proof. It is not hard to see that any element of $G$ is contained in some finite $V$-invariant subgroup which satisfies the conditions of the lemma. Thus it is sufficient to consider the case in which $G$ is finite. Let us prove (i). By [6, Lemma 2.1] we can assume that $G$ is a $p$-group for some prime $p$. By [15, Lemma 1.6] $G=G_{1} G_{2} \ldots G_{2^{n-1}}$ and by [7, Lemma 10.4.1] $G_{i}=J_{i} C_{G}(V)$. It follows from $G=[G, V]$ that $C_{G}(V) \subseteq G^{\prime} \subseteq$ $\Phi(G)$. So $G=\left\langle J_{i} ; 1 \leqq i \leqq 2^{n}-1\right\rangle$.

Now consider (ii). By the Feit-Thompson Theorem [5] $G$ is soluble. We shall prove the lemma by induction on the derived length of $G$. Suppose that for $\left[G^{\prime}, V\right]$ the assertion is true. Put $A=\bigcup_{i} J_{i}, B=A-\left(G^{\prime} \cap A\right), B_{i}=B \cap J_{i}$. Let $S$ denote the set of finite formal sequences of elements of $A$, i.e.

$$
S=\left\{\left(a_{1}, a_{2}, \ldots, a_{r}\right) ; a_{i} \in A\right\} .
$$

For each $s \in S$ define numbers $\alpha(s)$ and $\beta(s)$ as follows. Let $s=\left(a_{1}, a_{2}, \ldots, a_{r}\right)$. Then $\alpha(s)=\left|\left\{i ; a_{i} \in B\right\}\right|$. We put $\beta(s)=0$ if and only if for any $m \in\left\{1,2, \ldots, 2^{n}-1\right\} s$ contains at most one element of $B_{m}$. Otherwise, $\beta(s)=\min _{m}\left\{|i-j| ; i \neq j ; a_{i}, a_{j} \in B_{m}\right\}$. We denote by $\bar{s}$ the element $a_{1} a_{2} \ldots a_{r}$ of $G$. By (i) $G$ is generated by $A$ therefore the mapping $S \rightarrow G$ defined by $s \rightarrow \bar{s}$ is surjective. Let $h$ be an arbitrary element of $H=C_{G}(V)$. Then

$$
\begin{gathered}
\alpha(h)=\min _{s}\{\alpha(s) ; \bar{s}=h\} \\
\beta(h)=\min _{s}\{\beta(s) ; \bar{s}=h, \alpha(s)=\alpha(h)\} .
\end{gathered}
$$

We note that if $\alpha(h)=0$ then $h \in\left[G^{\prime}, V\right]$ and consequently $h$ belongs to $H_{0}=$ 
$\left\langle H \cap\left\langle J_{i}\right\rangle ; 1 \leqq i \leqq 2^{n}-1\right\rangle$. Let us show that $\beta(h)=0$ is possible only when $\alpha(h)=0$. Suppose that $\beta(h)=0$. Then there exists $s \in S$ such that $\bar{s}=h, \beta(s)=0$. Let $s=$ $\left(a_{1}, a_{2}, \ldots, a_{r}\right)$. Note that $h \in G^{\prime}$ as $G=[G, V]$. So in the factor group $G / G^{\prime}$ we have $b_{1} b_{2}, \ldots, b_{2^{n-1}}=1$ where $b_{m}=a_{k} G^{\prime}$ for $a_{k} \in B_{m}$. This immediately gives us $b_{1}=b_{2}=\cdots=$ $b_{2 n-1}=1$ which implies $\alpha(h)=0$.

Suppose that $\alpha$ is the least number such that $\alpha(h)=\alpha$ does not imply $h \in H_{0}$ and $\beta$ is the least number such that $\alpha(h)=\alpha$ and $\beta(h)=\beta$ does not imply $h \in H_{0}$. As we have shown above both $\alpha$ and $\beta$ are positive. Choose an element $h \in H$ such that $\alpha(h)=\alpha$, $\beta(h)=\beta$ and $h \notin H_{0}$. There exists a sequence $s=\left(a_{1}, a_{2}, \ldots, a_{r}\right) \in S$ such that $\bar{s}=h, \alpha(s)=\alpha$, $\beta(s)=\beta$. We have $a_{i}, a_{j} \in B_{m}$ where $j-i=\beta$. Denote by $s_{1}$ the initial segment of $s$ consisting of $j-2$ elements, by $s_{2}$ denote the final one consisting of $r-j$ elements. First suppose that $a_{j-1} \in J_{m}$. By [7, Lemma 10.4.1] $a_{j-1} a_{j}=b h_{1}$ where $b \in B_{m}, h_{1} \in H \cap\left\langle J_{m}\right\rangle$. Then $h h_{1}^{-1}=\bar{s}_{1} b h_{1} \bar{s}_{2} h_{1}^{-1}$. Clearly, $H \subseteq N_{G}\left(J_{k}\right)$ for $k=1,2, \ldots, 2^{n}-1$ so if $i=j-1$ then $\alpha\left(h h_{1}^{-1}\right) \leqq \alpha-1$. If $i \neq j-1$ then $\beta\left(h h_{1}^{-1}\right) \leqq \beta-1$. In any case under our assumptions $h h_{1}^{-1} \in H_{0}$, whence $h \in H_{0}$.

Let now $a_{j-1} \notin J_{m}$. By Lemma $4(i)$ there exist $m_{1}, m_{2} \in\left\{1,2, \ldots, 2^{n}-1\right\}$ such that $a_{j}^{-1} a_{j-1} a_{j}=c d c$ for suitable elements $c \in J_{m_{1}} \cap G^{\prime}$ and $d \in J_{m_{2}}$. Therefore $h=\bar{s}_{1} a_{j} c d c \bar{s}_{2}$, whence we get that $\beta(h) \leqq \beta-1$. Lemma 5 is now established.

Lemma 6. Suppose the hypotheses of Lemma 4 hold. Then for any element $x$ of $[G, V]$ there exist elements $x_{1}, x_{2}, x_{3}$ such that $x_{i} \in\left\langle J_{i}\right\rangle$ and $x=x_{1} x_{2} x_{3}$.

Proof. By [8, Lemma 4] there exist elements $y_{1}, y_{2}, y_{3}, h$ such that $x=y_{1} y_{2} y_{3} h$; $y_{i} \in J_{i} ; h \in C_{G}(V)$. By the preceding lemma $h=h_{1} h_{2} h_{3}$ for $h_{i} \in C_{G}(V) \cap\left\langle J_{i}\right\rangle$. Put $x_{1}=$ $y_{1} h_{1}, x_{2}=h_{1}^{-1} y_{2} h_{1} h_{2}, x_{3}=h_{2}^{-1} h_{1}^{-1} y_{3} h_{1} h_{2} h_{3}$. Then $x_{i} \in\left\langle J_{i}\right\rangle$ and $x=x_{1} x_{2} x_{3}$.

Lemma 7. With the hypotheses of Lemma 4 assume that $G=[G, V]$ and $R$ is a normal $V$-invariant subgroup of $G$ such that $R \cap C_{G}(V)=1$. Then $R$ possesses $a G V$-invariant series all of whose quotients are abelian.

Proof. Suppose that $R$ has no non-trivial abelian subgroup which is normal in $G V$. By [14, Lemma 2.2] there exists an element $a \in R \cap G_{i}$ such that $\left\langle a^{G}\right\rangle \cap C_{G}(a) \neq\left\langle a^{G}\right\rangle \cap$ $G_{i}$ for some $i \in\{1,2,3\}$. We assume that $a \in G_{1}$. Put $R_{i}=R \cap G_{i}, T=\left\langle a^{G}\right\rangle, T_{i}=T \cap G_{i}$, $D=C_{T}(a), D_{i}=D \cap G_{i}, 1 \leqq i \leqq 3$.

Evidently,

$$
\left\langle J_{i}\right\rangle \subseteq C_{G}\left(R_{i}\right)
$$

So $D_{1}=T_{1}$. By the choice of $a$ we can assume that $D_{2} \neq 1$. Put $K=C_{T_{1}}\left(D_{2}\right)$, $L=C_{T_{3}}(K)$.

Let $x$ be an arbitrary element of $G$. By Lemma $6 x=x_{1} x_{2} x_{3}$ where $x_{i} \in\left\langle J_{i}\right\rangle$. We have $a^{x}=a^{x_{2} x_{3}}$. By Lemma 4(i) $x_{2}^{-1} a x_{2}=c a_{1} c$ where $c \in J_{3}, a_{1} \in J_{1}$. By $\left(^{*}\right) a^{x_{2}} \in C_{T}\left(D_{2}\right)$, whence $a_{1} \in K, c \in C_{T}\left(D_{2}\right)$. Again by $\left({ }^{*}\right) x_{3} \in C_{G}(c)$, therefore $\left(c a_{1} c\right)^{x_{3}}=c a_{1}^{x_{3}} c$. By Lemma 4(i) $a_{1}^{x_{3}}=b a_{2} b$ where $b \in J_{2}, a_{2} \in J_{1}$. We note that $a_{1}^{x_{3}} \in C_{T}(L)$, whence $b, a_{3} \in C_{T}(L)$. This argument shows that $L \subseteq Z(T)$. Suppose that $L=1$. Then $v_{3}$ acts fixed-point-freely on $C_{T}(K)$ which gives us that $T_{1} \subseteq C_{G}\left(D_{2}\right)$, that is $K=T_{1}$. 
Consequently, $D_{2} \subseteq C_{G}\left(\left\langle T_{1}, T_{2}\right\rangle\right)$. By [14, Lemma 1.5, Corollary 1] $\left\langle T_{1}, T_{2}\right\rangle=\left[T, v_{3}\right]$. So

$$
D_{2} \subseteq C_{G}\left(\left[T, v_{3}\right]\right)
$$

Evidently,

$$
G_{3} \subseteq N_{G}\left(\left[T, v_{3}\right]\right) \text {. }
$$

Now let $y$ be an arbitrary element of $G$. Again by Lemma $6 y=y_{1} y_{3} y_{2}$ where $y_{i} \subseteq\left\langle J_{i}\right\rangle$. We have $a^{y_{1}}=a$. Using $\left({ }^{* *}\right)$ and $\left({ }^{* * *}\right)$, we get $D_{2} \in C_{G}\left(a^{y^{3}}\right)$. By $\left(^{*}\right)\left[D_{2}, y_{2}\right]=1$, whence $D_{2}=D_{2}^{y_{2}} \subseteq C_{G}\left(a^{y_{3} y_{2}}\right)=C_{G}\left(a^{y}\right)$. Therefore $1 \neq D_{2} \subseteq Z(T)$. This contradicts the assumption that $R$ has no non-trivial abelian subgroup which is normal in $G V$. The lemma is established.

\section{Proof of theorem}

Let $C=C_{G}(V)$ be Chernikov, and let $S$ be some maximal 2-subgroup of $G$ containing $V$. By [13, Lemma 3.1] $S$ is Chernikov. Let $F$ be the minimal subgroup of finite index in $S$. We denote by $Q$ the maximal normal 2 '-subgroup of $G$. First, let us prove that $[Q, V]$ is hyperabelian. By Lemma 2 (i) it suffices to show that $[Q, V]$ has a nontrivial normal $V$-invariant abelian subgroup. Suppose that any such subgroup of $[Q, V]$ is trivial. Then by Lemma 7 each normal $V$-invariant subgroup of $[Q, V]$ has non-trivial intersection with $C$. In this case $[Q, V]$ contains a minimal non-trivial normal $V$-invariant subgroup $M$. By a theorem of McLain [13, p. 11] $M$ is abelian and we obtain a contradiction. Thus $[Q, V]$ is hyperabelian. Therefore in order to prove (i) it suffices to show that $[Q, V]$ contains a subgroup $R$ such that $R$ is normal in $G$ and $G / R$ is Chernikov.

Let $v_{1}, v_{2}, v_{3}$ be the involutions of $V, G_{k}=C_{G}\left(v_{k}\right), F_{k}=F \cap G_{k}, Q_{k}=Q \cap G_{k}, J_{k}=$ $\left\{x \in G_{k} ; x^{v_{i}}=x^{-1}\right.$ for $\left.i \neq k\right\} ; 1 \leqq k \leqq 3$. Then $F=F_{1} F_{2} F_{3}$ [3, Lemma 6] and $F_{k}=C_{F}(V)$ $\left(F \cap J_{k}\right)$. We note that $\left(F \cap J_{i}\right)\left(F \cap J_{j}\right) \cap\left(F \cap J_{k}\right)=1$ implies that $F \cap J_{k}$ is divisible. By Lemma 5 (i) $[Q, V]=\left\langle J_{k} \cap Q ; 1 \leqq k \leqq 3\right\rangle$. Let $x$ be an element of $F \cap J_{k}$ for some $k \in\{1,2,3\}$ and $y$ an element of $J_{j} \cap Q$ for some $j \in\{1,2,3\}$. If $j \neq k$, then $v_{k}$ inverts $y^{x}$ whence $y^{x} \in\left[Q, v_{k}\right] \subseteq[Q, V]$. Suppose $j=k$. Then by Lemma $3, y^{x}=y$. Thus $F \cap J_{k}$ normalizes $[Q, V]$. Evidently so does $Q C_{F}(V)$ and we have $Q F \subseteq N=N_{G}([Q, V])$. This gives us [13, Theorem 3.17] that $N$ is of finite index in $G$. Since by Lemma 2(iii) $Q /[Q, V]$ is Chernikov we have that $N /[Q, V]$ is Chernikov. So $R=\bigcap_{x} x^{-1}[Q, V] x$ is normal in $G$ and $G / R$ is Chernikov.

Let us now assume that $C_{G}(V)$ is finite. Then by Lemma 2 (iii) $[Q, V]$ has finite index in $Q$. Since $[Q, V]$ is hyperabelian, by Lemma $1 Q V$ is hyperabelian. Let $r=\operatorname{rank}(F)$. We prove by induction on $r$ that $Q F V$ is hyperabelian. If $r=0$ then $F=1$ and $Q F V=Q V$ is hyperabelian. Let $r \geqq 1$. Since $F=F_{1} F_{2} F_{3}[3$, Lemma 6] without loss of generality we can assume that $\operatorname{rank}\left(F_{2} F_{3}\right) \leqq r-1$ and consequently by induction $Q F_{2} F_{3} V$ is hyperabelian. If $Q F V$ is not hyperabelian then there exists a quotient $T$ of $Q F V$ such that $T$ does not possess a non-trivial normal abelian subgroup. For the sake of simplicity we 
assume that $T=Q F V$. Let $B$ be a non-trivial abelian subgroup of $Q$, which is normal in $Q F_{2} F_{3} V$. Put $B_{1}=B \cap G_{1}$. If $B_{1}=1$ then $B \subseteq Z\left(\left[Q, v_{1}\right]\right)$. By Asar's result [1] $Q /\left[Q, v_{1}\right]$ is soluble so $B$ centralizes some term of the derived series of $Q$. This shows that $Q$ possesses a non-trivial characteristic abelian subgroup and we obtain a contradiction. Let $B_{1} \neq 1$. By Lemma $3 F_{1} \subseteq C_{G}\left(B_{1}\right)$. whence $\bigcap_{x \in F_{1}} x^{-1} B x$ is a non-trivial abelian subgroup which is normal in $Q F V$. This contradicts our assumptions and proves that $Q F$ is hyperabelian. As $Q F$ is of finite index in $G$ [13, Theorem 3.17], by Lemma $1 G$ is hyperabelian. The proof is now completed.

\section{REFERENCES}

1. A. O. Asar, Locally finite groups with Chernikov centralizers, J. Algebra 68 (1981), 170-176.

2. A. O. Asar, The solution of a problem of Kegel and Wehrfritz, Proc London Math. Soc. (3) 45 (1982), 337-364.

3. V. V. Belyaev, Locally finite groups with Chernikov Sylow p-subgroups, Algebra i Logika 20 (1981), 605-619 (Russian).

4. S. N. Chernikov, On periodic groups of automorphisms of extremal groups, Mat. Zametki 4 (1968), 91-96 (Russian).

5. W. Feit and J. G. Thompson, Solvability of groups of odd order, Pacific J. Math. 13 (1963), 775-1029.

6. D. Goldschmidt, Weakly embedded 2-local subgroups of finite groups, J. Algebra 21 (1972), 341-351.

7. D. GoRenstein, Finite Groups (Harper and Row, New York, 1968).

8. D. Gorenstein and J. H. Walter, On finite groups with dihedral Sylow 2-subgroups, Illinois J. Math. 6 (1962), 553-593.

9. B. Hartley, Periodic locally soluble groups containing an element of prime order with Chernikov centralizer, Quart. J. Math. Oxford (2) 33 (1982), 309-323.

10. B. Hartley, Centralizers in locally finite groups, in Proceedings, Group Theory Conference (Bressanone, 1986).

11. B. Hartley, Fixed points of automorphisms of certain locally finite groups and Chevally groups, J. London Math Soc. (2) 37 (1988), 421-436.

12. L. G. Kovacs and G. E. WALL, Involutory automorphisms of groups of odd order and their fixed point groups, Nagoya Math. J. 27 (1966), 113-120. 1973).

13. O. H. Kegel and B. A. F. Wehrfritz, Locally Finite Groups (North-Holland, Amsterdam,

14. P. Shumyatsky, Periodic groups with a regular four-group of automorphisms, Izv. Vuzov. Matematika 11 (1987) 78-82 (Russian).

15. P. Shumyatsky, Groups with regular elementary 2-groups of automorphisms, Algebra $i$ Logika 27 (1988), 715-730 (Russian).

Department of Mathematics

Technion-Israel Institute of Technology

HAIFA 32000

ISRAEL 